\title{
Automated Detection of Retinal Hemorrhage based on Supervised Classifiers
}

\author{
KA Sreeja ${ }^{1}$, SS Kumar ${ }^{2}$ \\ ${ }^{1}$,Department of Electronics and Communication Engineering. SCMS School of Engineering and Technology, \\ Karukutty, Ernakulam. Kerala, India \\ ${ }^{2}$ Department of Electronics and Instrumentation Engineering. NICHE, Thuckalay. Tamilnadu, India
}

\begin{tabular}{l}
\hline \hline Article Info \\
\hline Article history: \\
Received May 28, 2019 \\
Revised Feb 28, 2020 \\
Accepted Mar 6, 2020 \\
\hline
\end{tabular}

\section{Keywords:}

Retinal hemorrhage Diabetic retinopathy Fundus image Splat feature classification GLCM features

\section{Corresponding Author:}

Sreeja K.A, Department of Electronics and Communication Engineering, SCMS School of Engineering and Technology, Karukutty, Ernakulam, Kerala, India.

Email: ka.sreeja@gmail.com

\begin{abstract}
Supervised machine learning algorithm based retinal hemorrhage detection and classification is presented. For developing an automated diabetic retinopathy screening system, efficient detection of retinal hemorrhage is important. Splat, which is a high level entity in image segmentation is used to mark out hemorrhage in the pre-processed fundus image. Here, color images of retina are portioned into different segments (splats) covereing the whole image. With the help of splat level and GLCM features extracted from the splats, three classifiers are trained and tested using the relevant features. The ground-truth is established with the help of a retinal expert and using dataset and clinical images the validation was done. The output obtained using the three classifiers had more than $96 \%$ sensitivity and accuracy.
\end{abstract}

Copyright (C) 2019Institute of Advanced Engineering and Science. All rights reserved.

\section{INTRODUCTION}

The World Health Organisation estimated that by 2030, there will be nearly 366 million people with Diabetic mellitus (DM) [1]. A microvascular complication of DM that is responsible for a major share of cases of blindness in the world is the Diabetic Retinopathy (DR). The severe complications like Microaneurysms, Exudates, Occlusion, hemorrhages, etc., together known as DR. The DR becomes more severe when it goes undetected for a long period of time. Retinal hemorrhages and other symptoms are usually diagnosed by an ophthalmoscope or a fundus camera that are capable to examine inside of eye. In order to reduce the diagnosing time, human error and increase the accuracy, several algorithms have been developed for the early detection of DR and all of them use machine learning techniques. An expert, usually an ophthalmologist or a retinal specialist provides the ground truth to train the system. The pre-processed fundus image features are extracted and applied to a supervised classifier which is trained with the relevant features by feature subset selection. In this paper, classification of hemorrhage and non-hemorrhage fundus images carried out using three different classifiers is presented. The techniques used to develop the algorithm is based on recent researches.

When compared to large hemorrhages, it is seen that small hemorrhages are irregular in shape. Many systems have been developed to find these abnormalities. DR detection based on Convolutional Neural Network $(\mathrm{CNN})$ using binocular fundus images and its correlation is suggested by Zeng et.al [2]. The model has a Siamese-like architecture that predicts the possibility of DR for both the eyes by correlating the pathological as well as the physiological condition of eyes. In our work one of the classifier decision is based on Neural network (NN). D Kumar et.al. [3] presented a radiomics-driven Computer Aided Diagnosis (CAD) based method. In order to overcome the limitations with current CAD approaches such as decision making a CLass-Enhanced Attentive Response Discovery Radiomics CLEAR-DR is proposed to aid clinical diagnosis of DR. Another important symptom of diabetic retinopathy is exudates, which are similar to hemorrhage pixels. 
An Early detection of exudates is presented byWisaeng and Sa-Ngiamvibool [4] using morphology mean shift algorithm (MMSA). After pre-processing, the image undergoes a coarse segmentation using mean shift and classification is done using the mathematical morphology algorithm (MMA). Detection of bright and dark lesion which can be hemorrhages or exudates, using a combination of matched filter response(MFR) and Laplacian of Gaussian Response (LoG) [5] produced a 96.10\% - 96.99\% accuracy for various publically available database in hemorrhage detection. Multi-resolution analysis(MRA) is given importance in the work done by Lahmiri [6]. The statistical features obtained after MRA is fed to a support vector machine to grade retinal hemmorhage. Detection of hemorrhage pixels from the bright optical disc is always a constraint. Many methods are already prevailing in order to remove optical disc from the fundus image. Five optic disc detection methods with an algorithms committee having waited voting is presented by Silva et.al. [7] where, six public benchmark databases with a total of 1566 images is employed. Even though, in our work the optical disc is not removed, this method is useful when pixel based approach is considered. One such method of optic disc removal is used in exudate detection that involves mathematical morphology [8]. After morphological operation, the hard exudates are extracted using adaptive fuzzy logic.

The purpose of this research is to develop a supervised classification model using three different classifiers and compare the output based on their sensitivity, specificity and accuracy. Retinal hemorrhages are demarcated with the help of an ophthalmologist who use a high-level representation entity known as splat [9]. Splats are a collection of pixels that have similar fundamental features. A two-step feature selection process is carried out to remove redundant features from the splat and these features are applied to a supervised classification to predict the possibility of hemorrhage splats in the whole image. The hemorrhage is finally detected and shown as bright spots on the dark oponency image. All the three classifiers are tested and their responses are tabulated. Section 2 describes the research method. Feature extraction and classification are portrayed in this section. Section 3 gives the result and discussion and section 4 concludes the work

\section{RESEARCH METHOD}

Pixels that are assumed to have similar spatial location and share same structural features such as color and intensity are partitioned into non-overlapping splats and spread over the entire image.[10]

Splat based method uses several re-sampling strategies. As multiple sampling is performed, the background region consists of few number of larger splats whereas the foreground region consists of a large number of smaller splats. In a fundus image with hemorrhage, the total number of hemorrhage pixels is comparatively less when the entire image is considered [11]. Therefore, a splat based method is more likely to have better diversity in training the samples.

Splats are generated using watershed segmentation algorithm[10]. Splats are created in such a way that it preserves the boundaries between hemorrhage pixels and retinal background. In order to create meaningful splats, a scale specific over segmentation is performed. This is done in two steps. At first the gradient magnitude of contrast enhanced dark-bright opponent image is taken using different scales. It is done because of the variability in appearance of hemorrhages. All these values are aggregated and the maximum of the gradient value with its scale of interest(SOI) is taken to perform watershed segmentation [12].

The gradient magnitude is computed using the equation

$$
|\nabla I(x, y ; s)|=\sqrt{I_{x}(x, y ; s)^{2}+I_{y}(x, y ; s)^{2}}
$$

where $I(x, y ; s)$ is the image. Now establishing a scale-space representation of the image using Gaussian kernels $G_{s}$, the gradient magnitude is calculated from its horizontal and vertical derivatives as

$$
\begin{aligned}
& |\nabla I(x, y ; s)|=\sqrt{\left[\frac{\partial}{\partial x}\left(G_{s} * I(x, y)\right)\right]^{2}+\left[\frac{\partial}{\partial y}\left(G_{s} * I(x, y)\right)\right]^{2}} \\
& |\nabla I(x, y ; s)|=\sqrt{\left.\left.\left[\frac{\partial G_{s}}{\partial x} * I(x, y)\right)\right]^{2}+\left[\frac{\partial G_{s}}{\partial y} * I(x, y)\right)\right]^{2}}
\end{aligned}
$$

where the symbol $*$ denotes convolution and $\frac{\partial G_{S}}{\partial x}$ and $\frac{\partial G_{S}}{\partial y}$ are the first order derivatives of Gaussian in the horizontal and vertical direction using the scale $\mathrm{s}$

The maximum of the gradient magnitude is

$$
|\nabla I(x, y)|=\max _{i}\left|\nabla I\left(x, y ; s_{i}\right)\right|
$$


Since the topographic surface in watershed algorithm is important [11]to obtain genuine splats, the maximum of the gradient magnitude is taken for certain scale of Interest (SOI). Splats created using different scales exploiting the same watershed algorithm is shown in the Fig. 1.

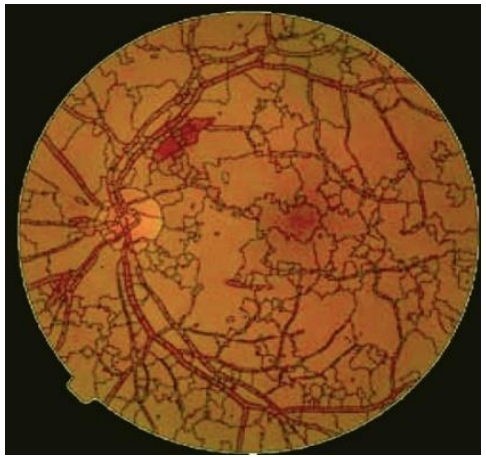

(a)

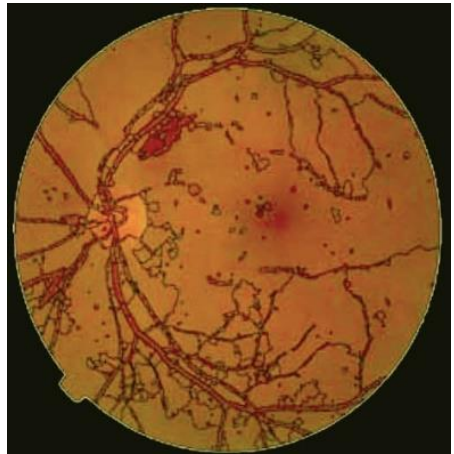

(b)

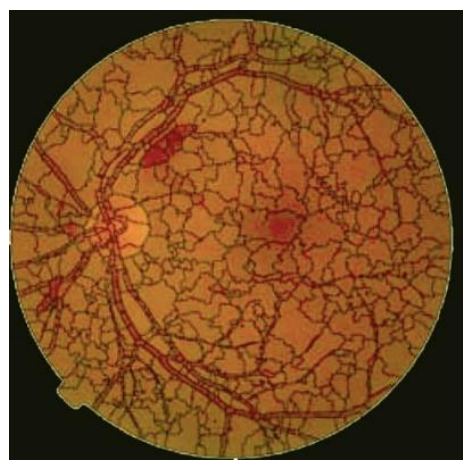

(c)

Figure 1. Splats created using different scales.

Scale band taken for Fig. 1(a) is most accurate when compared to scales used in Fig. 1(b) and Fig. 1(c) as it comes under the desired SOI for hemorrhage detection. The retinal background is represented by larger splats and blood regions are represented as smaller splats Fig. 1(a). The scale in Fig. 1(c) is suitable in removal of larger areas such as optic disc. The scale used in Fig. 1(b) is a fine scale and also not in the desired SOI. Again this scale range can be used in the detection of Microaneurysms. The number of splats is kept under a certain limit or threshold to achieve speed without much compromising the accuracy.

\subsection{Splat Based Labelling by ophthalmologist}

The samples taken from watershed algorithm are labelled by experts as the supervised algorithms are to be labelled using the MATLAB software. Small hemorrhages shown as purple dots, are indicated by a single point in Fig. 2.

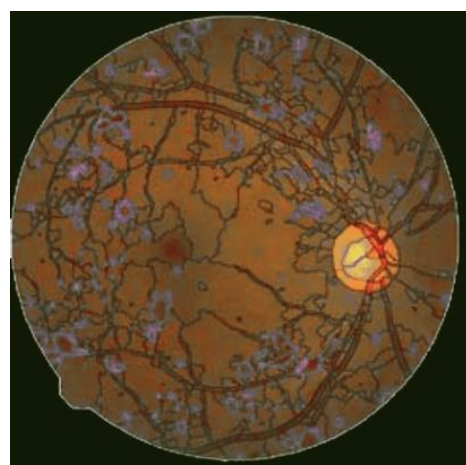

Figure 2. Small hemorrhages noted as purple dots.

The expert performs a single click on where hemorrhage is spotted and the pixel values are noted down. The images that contain hemorrhages are given a label of ' 1 ' and the rest are given as ' 0 '. The splats are designed to preserve hemorrhage boundaries, and the reference standard result obtained is less noisy. 


\subsection{Feature Extraction from Splats}

After assigning reference labels for splats, a classifier can be trained to detect the target objects. An altogether of 352potentially relevant features are taken to train the classifiers. They are:

1) Color: Colors ofeach splat is extracted in RGB color space and dark-bright (db), red-green (rg), and blue-yellow (by) opponency images [13], which comes to six colour components.

2) Difference Of Gaussian(DoGFilter): Difference of Gaussian (DoG) kernels are applied at five differentsmoothing scales with one baseline scale in order to take advantage of Gaussian scale space[14][15].

3) Responses from GaussianFilter Bank[13]: A Gaussian filter bank which include a first orderderivative at two orientations and a second order derivative with three orientations are applied to the green channel.

4) Responses from SchmidFilter Bank: It has 13 kernels which are rotationally invariant and is applied to the dark bright opponent image.

5) Responses from Local Texture Filter Banks: This filter bank contains local entropy filter, local range filter and local standard deviation filter which compute the entropy, standard deviation and intensity range of one pixel in a given region [16]

The above features are aggregated to obtain a meaningful response image which has low inter splat similarity and high intra splat similarity[13]-[19].The features mentioned are pixel- based responses. In addition to these features, we take splat wise features according to Gray-Level Co-occurrence Matrix (GLCM)[16]-[22]statistics. These are splat area, extent, texture, solidity and orientations. The complete feature set is shown in Table 1

\subsection{Preliminary Feature Selection and classification}

A two-step feature selection method is taken here so as to take only the relevant features and discard the irrelevant and redundant ones[23]. The preliminary feature selection is done using a filter approach in order to eliminate the features that are immaterial in discriminating hemorrhage and non-hemorrhage splats. A quadratic discriminant analysis(QDA)[24]is performed and by inspecting the features' variation with misclassification error(MCE)[25]. The preliminary features are chosen when the smallest MCE is reached.

Table 1. The complete feature set.

\begin{tabular}{lll}
\hline Features & Number & Description \\
\hline Shape Features & & \\
\hline Colour & $6 \times 4$ & RGB and dark-bright (db), red-green(rg), blue-yellow(by) opponency[13] \\
DoG filter bank & $30 \times 4$ & RGB and db, red-green rg, blue-yellow by[15] \\
Gaussian Filter Bank & $30 \times 4$ & First order and second order derivatives in horizontal and vertical plane. \\
Schmid filter bank & $13 \times 4$ & 13 kernels [17] \\
Local Texture filter & $3 \times 4$ & Local range, standard deviation and entropy of a pixel in given neighborhood[16] \\
\hline GLCM Features & & \\
\hline Splat area & 1 & Number of pixels in splat \\
Splat extent & 1 & Proportion of pixels in bounding box that are also in splat \\
Splat orientation & 1 & Angle between horizontal and major axis of the ellipse having same second- moments as splats. \\
Splat Solidity & 1 & Proportion of pixels in convex hull that are also in splat. \\
Texture & 4 & Statistics of GLCM: Contrast, correlation, energy and homogeneity [16] \\
Tamura Signatures & 3 & Coarseness, directionality and contrast of dbopponency associated with each splat[20] \\
Edge Strength ratio & 6 & Ratio of maximum and minimum edge strength between neighboring splats. \\
Vignetting artifacts & 1 & Closest distance of splat centroids to boundaries of FOV. \\
Vessel probability & 1 & Vessel probability map averaged within splats using vessel segmentation algorithm [21] \\
Impact of anatomical & 2 & Distance of automatically detected optic disc and fovea to splat centroids [22] \\
structures & &
\end{tabular}

After preliminary selection, a wrapper approach is performed in order to get an optimal combination of relevant features with minimum redundancy. It is the peculiarity of the wrapper approach that it assesses different combinations of feature subsets customized for a certain classification algorithm with higher computation time.The combinations are evaluated using a kNN Classifier. All the selected features are now applied to a sequential forward feature selection subset(SFS).

\subsection{Classification using different classifiers}

After feature selection, threedistinct trained classifiers are set up with the set of features and reference label instances. 


\section{A. kNN Classification}

The kNN algorithm assigns soft class labels. The two classes defined or the outputs are hemorrhage splat or non-hemorrhage splat. The classifier decides the class of a particular splat based on the Euclidean distance of the features in an optimized feature space. As the value of $\mathrm{k}$ is increased the computation time increases and the splats are more accurately identified. But since all the k nearest neighbors are not near, an optimum value of $\mathrm{k}$ is chosen instead of an arbitrary value.

\section{B. SVM Classification}

Support Vector Machines include the concept of hyperplanes to distinguish between classes. The features are transformed to the required form using a linear kernel. The features were optimized and only the relevant number of features were trained to the classifier. A least squares SVM classifier with Radial Basis Function (RBF) Kernel is used here[26]. The RBF Kernel function is defined as

$$
K\left(x, x^{\prime}\right)=\exp \left(\frac{\| x^{\prime}-x||}{2 \sigma^{2}}\right)^{2}
$$

Where $\mathrm{x}$ and $\mathrm{x}$ ' are two feature vectors and $\sigma$ is a free parameter and ||$x^{\prime}-x \|^{2}$ is taken as squared Euclidean distance parameter.

\section{ANN Classification}

ANNs rely up on the concept of artificial neurons which is biologically inspired based on the function of brain. Here each neurons or nodes are held within a layer. The Neural Network consists of input layers, hidden layers and a transfer function or a threshold function. All the nodes are interconnected and they form a network. The nodes are trained using backpropagation algorithm. The nodes are trained by reducing their error through several iterations. The input to the ANN classifier is the relevant feature set and they are transformed using the desired weights from the hidden layer. Finally using a Sigmoid transfer function the output class is determined.

\section{RESULTS AND DISCUSSION}

\subsection{Data Collection and Pre-processing}

Images were acquired from two sources. One from the publically available database DIARETDB1(http://www.it.lut.fi/project/imageret/diaretdb1/index.html) and the second set of clinical images from Dr. Bhejan Singh's eye hospital solely for educational and research purpose. The clinical image was captured using a "Remidio Non-Mydriatic Fundus On Phone (FOP-NM10)" Camera with an FOV of $40^{\circ}$, working distance of $33 \mathrm{~mm}$ and an ISO range from ISO 100 to 400 . A total of 1500 images were taken 1050 for training, 225 images for testing and 225 for validation. The reference standard observations were accomplished by an ophthalmologist expert using the splat-based interpretation. Overall 1200(950 from training set 150 from testing) images were marked by the expert from a total of 1500 . Preprocessing is done in order to adapt the colour variation throughout the dataset and also to equalize the intensity of the image. Histogram equalization is done using Contrast limited Adaptive Histogram Equalization(c)[27].Also Each image is normalized according to its prevailing pixel value at the three colour channels. The pixel values that occur frequently are shifted to the beginning of RGB colour space.

\subsection{Feature Subset selection}

From the 1050 training images. 10500 splats were created among which 300 are hemorrhage splats. This counts to a very low number of hemorrhage splat density. So images with at least 6 splats are taken for training, where 6 is arbitrarily chosen. After sequential forward feature selection subset(SFS) only the relevant features were considered whereas the insignificant and redundant ones were removed from the feature set.

The final feature set consists of 50 features from the 352 features obtained by filter approach and from this set 19 features were finally obtained by wrapper approach. The details of the final selected features are given in Table 2.

Table 2. The details of final selected features.

\begin{tabular}{lll}
\hline Features & Number & Description \\
\hline DoG filter bank & $\mathrm{s} 2-\mathrm{s} 0.5$ & from Green channel \\
$\begin{array}{l}\text { DoG filter bank } \\
\text { DoG filter bank }\end{array}$ & $\mathrm{s} 4-\mathrm{s} 0.5$ & $\mathrm{from}$ db and rg opponency \\
faussian Filter Bank & $\mathrm{s}=8$ orientation: 2,3 & Mean of second order Gaussian derivative from green channel \\
& $\mathrm{s}=1,2,4$ & Mean of second order Gaussian derivative from green channel \\
orientation: $1,2,3$ & \\
& response $=11$ & from db opponency \\
fchmid filter bank & $\mathrm{s}=8,16$ & from Green channel \\
\hline
\end{tabular}




\subsection{Classification of splats using different classifiers}

The splats are represented as a 19 dimensional feature vector and each classifier is trained based on these features.

\section{a. kNN Classifier}

Different values of $\mathrm{k}$ were tested whose values are chosen between 15 to 160 that involves both feature selection as well classification. After repeated iterations, the value of $\mathrm{k}$ was fixed at 105 without compromising the computation time and prediction accuracy.

The algorithm was validated using the image from the dataset DIARETDB1 and the ROC curve was plotted with an AUC of 0.94

\section{b. SVM Classifier}

The features were optimized and only the 19 relevant features were trained to the classifier. A least squares SVM classifier with Radial Basis Function (RBF) Kernel is used here. The confusion matrix for the hemorrhage splat detection is shown in Table 3.

Table 3. The confusion matrix for SVM classifier

\begin{tabular}{c|cc|c}
\hline $\mathrm{n}=10500$ & $\begin{array}{c}\text { Predicted } \\
\text { NO }\end{array}$ & $\begin{array}{c}\text { Predicted } \\
\text { YES }\end{array}$ & \\
\hline Actual NO & $\mathrm{TN}=5250$ & $\mathrm{FP}=139$ & 5389 \\
Actual YES & $\mathrm{FN}=621$ & $\mathrm{TP}=4490$ & 5111 \\
\hline & 5871 & 4629 & \\
\hline
\end{tabular}

\section{c. ANN Classifier}

The input to the ANN [28] classifier is the 19 feature set and they are transformed using the desired weights from the hidden layer. Finally using a sigmoid transfer function, the output class is determined. The network protocols used for detection of hemorrhage class from the splats is given in Table 4.

Table 4. The protocols used for detection of hemorrhage class.

\begin{tabular}{ll}
\hline Features & Hemorrhage splats \\
\hline Learning rule base & Delta rule \\
Transfer function & Sigmoid \\
Hidden Layer Elements & 30 \\
Preprocessing filter & Feature set \\
Number of training iterations & 1000 \\
Training algorithm & Bayesian \\
Neural network & Fitting network \\
Training time- Core i5, $4.10 \mathrm{GHz}$ & 10 min \\
Number of training splats & 7350 \\
Number of testing splats & 3150 \\
\hline
\end{tabular}

The hemorrhage splats were successfully detected using the three classifiers and the result of a fundus image along with its receiver operator characteristics are shown in Figs.3. Splat based ROC curves corresponding to fundus image using the three classifiers is shown in Fig. 4. The AUC for ANN Classifier was 0.96 for SVM it was 0.94 and using the $\mathrm{kNN}$ classifier was 0.93 . The best operating point on the curve for different classifiers are shown in Table 5.

In this manuscript, a set of features are extracted from each splat for defining its characteristic properties. In a selected feature space, these splats are taken as supervised classification samples. Splat based image annotations makes it easier for ophthalmologists for modeling unevenly shaped abnormalities in images. Further, it is resistant to intensity bias and noise. Analyzing how the performance of a DR detection system is related to the detection of rare large hemorrhages is quite interesting and challenging. It is found that the unweighted performance metrics like AUC or sensitivity and specificity will not be affected if we integrate the present hemorrhage detection system at a suitable threshold level. The reason is that, only the presence or absence of DR is usually indicated by the binary reference labels. In this approach, color images of retina are partitioned into different non-overlapping segments (splats). A set of features are extracted from each splat this inturn help us to detect retinal hemorrhage. 


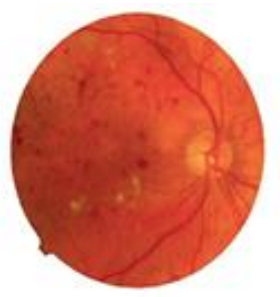

(a)

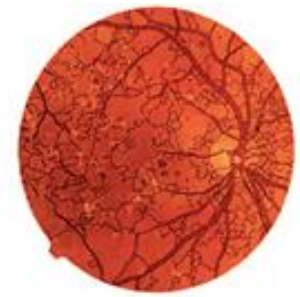

(b)

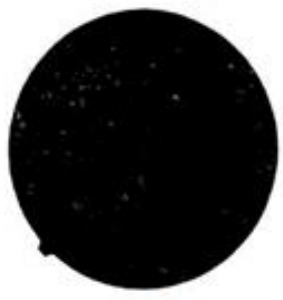

(c)

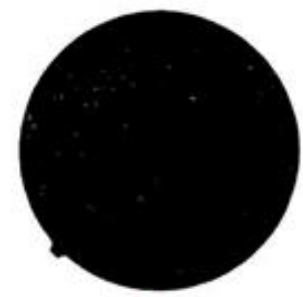

(d)

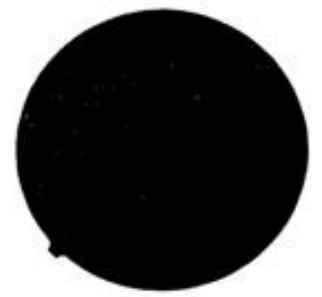

(e)

Figure 3. Hemorrhage splats detection: (a) Input image; (b) Image enhanced and splats generated; (c)-(e) Hemorrhage classification using ANN, SVM and kNN respectively.

The corresponding ROC curve for the input image is given in Fig. 4.

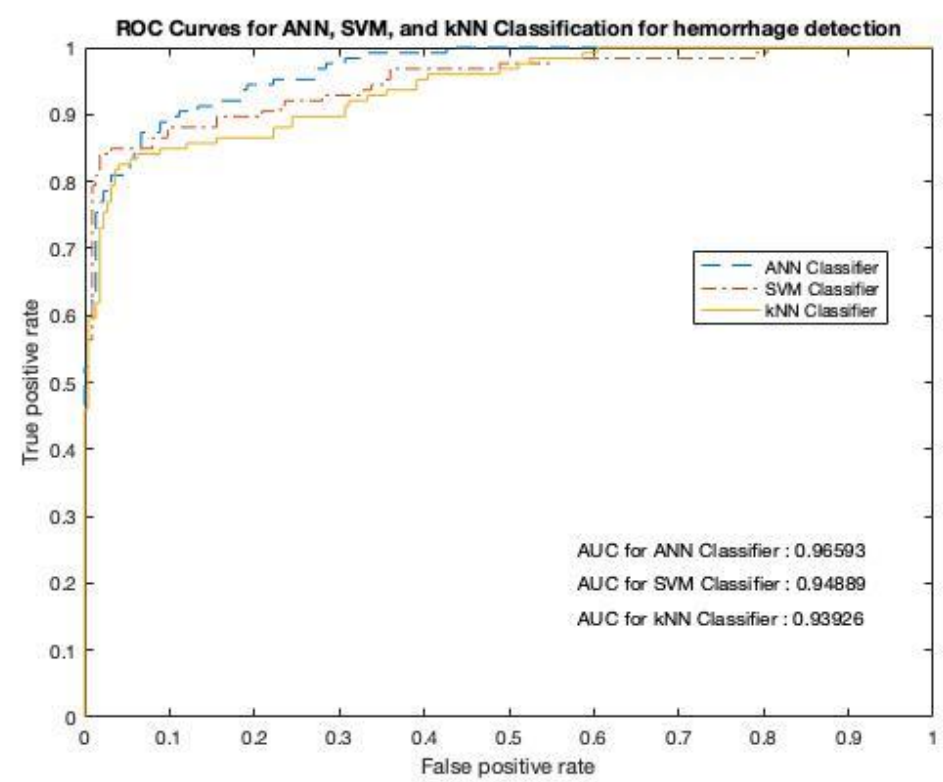

Figure 4. ROC curves for ANN, SVM and kNN classification for hemorrhage detection.

Table 5. The best operating point on the curve for different classifiers

\begin{tabular}{ccc}
\hline Classifier & Sensitivity & Specificity \\
\hline ANN & 0.96 & 0.67 \\
SVM & 0.94 & 0.44 \\
kNN & 0.93 & 0.42 \\
\hline
\end{tabular}

Superior results were obtained using ANN classifier when compared with other classifiers such as SVM and kNN classifiers. All the classifiers are proven to detect retinal hemorrhages with very high accuracy and since different splats in the image represents a large feature space, the neural network classifiers can outperform other classifiers. This was one of the reason to take ANN classifier into consideration. While other classifiers such as SVM and kNN are proven to provide superior results. When compared with the work of [29] the method has proved a sensitivity of 0.96 using ANN classifier against the sensitivity of 0.82 with kNN classification used in [29]. Compared with the work presented in [30], where fuzzy logic is used for classification, this method has proven superior results with a sensitivity of 0.96 against 0.86 . 


\section{CONCLUSION}

A splat based feature classification is presented for the detection of retinal hemorrhage. The proposed classification strategy can model different lesions with different texture size and appearance. The algorithm is validated on the publically available database DIARETDB1 and clinical image which was captured using a "Remidio Non-Mydriatic Fundus on Phone (FOP-NM10). The proposed method when compared with other methods in the literature suggest that it provides superior results when neural network classifier is being used. So the detection strategy can be incorporated into comprehensive DR assisting system for opthomologists.

\section{REFERENCES}

[1] World Health Organizat ion, P revent ion of blindness from diabet es mellit us, 2006.

[2] X. Zeng, H. Chen, Y. Luo, and W. Ye, "Automated Diabetic Retinopathy Detection Based on Binocular Siameselike Convolutional Neural Network," IEEE Access, vol. PP, no. c, pp. 1-1, 2019.

[3] D. Kumar, G. W. Taylor, and A. Wong, "Discovery Radiomics with CLEAR-DR: Interpretable Computer Aided Diagnosis of Diabetic Retinopathy," IEEE Access, vol. 7, no. c, pp. 25891-25896, 2019.

[4] K. Wisaeng and W. Sa-Ngiamvibool, "Exudates Detection Using Morphology Mean Shift Algorithm in Retinal Images," IEEE Access, vol. 7, no. c, pp. 11946-11958, 2019.

[5] S. S. Kar and S. P. Maity, "Automatic Detection of Retinal Lesions for Screening of Diabetic Retinopathy," IEEE Trans. Biomed. Eng., vol. 65, no. 3, pp. 608-618, 2018.

[6] S. Lahmiri, "High-frequency-based features for low and high retina haemorrhage classification," Healthc. Technol. Lett., vol. 4, no. 1, pp. 20-24, 2016.

[7] R. R. V. E Silva, F. H. D. De Araújo, L. M. R. Dos Santos, R. M. S. Veras, and F. N. S. De Medeiros, "Optic disc detection in retinal images using algorithms committee with weighted voting," IEEE Lat. Am. Trans., vol. 14, no. 5, pp. 2446-2454, 2016.

[8] N. G. Ranamuka and R. G. N. Meegama, "Detection of hard exudates from diabetic retinopathy images using fuzzy logic," IET Image Process., vol. 7, no. 2, pp. 121-130, 2013.

[9] L. Tang, M. Niemeijer, J. M. Reinhardt, S. Member, M. K. Garvin, and M. D. Abràmoff, "Splat Feature Classi fi cation With Application to Retinal Hemorrhage Detection in Fundus Images," IEEE Transactions on Medical Imaging, vol. 32, no. 2, pp. 364-375, 2013.

[10] J. Fairfield, "Toboggan contrast enhancement for contrast segmentation," $199010^{\text {th }}$ International Conference on Pattern Recognition, vol. i, pp. 712-716, 1990.

[11] N. V Chawla, N. Japkowicz, and A. Ko, "Editorial: Special Issue on Learning from Imbalanced Data Sets." SIGKDD Explor. Newsl. vol.6, pp.1-6. 2004. DOI: https://doi.org/10.1145/1007730.1007733

[12] Yung-Chieh Lin, Yu-Pao Tsai, Yi-Ping Hung, and Zen-Chung Shih, "Comparison between immersion-based and toboggan-based watershed image segmentation," IEEE Trans. Image Process., vol. 15, no. 3, pp. 632-640, Mar. 2006.

[13] M. D. Abra'moff et al., "Automated Segmentation of the Optic Disc from Stereo Color Photographs Using Physiologically Plausible Features,” Investig. Opthalmology Vis. Sci., vol. 48, no. 4, p. 1665, Apr. 2007.

[14] B. M. Romeny, Front-end vision and multi-scale image analysis: multi-scale computer vision theory and applications, written in Mathematica. 1st ed., Springer, 2003.

[15] L. Tang, M. Niemeijer, and M. D. Abramoff, "Splat feature classification: Detection of the presence of large retinal hemorrhages," 2011 IEEE International Symposium on Biomedical Imaging: From Nano to Macro, pp. 681-684, 2011.

[16] O. Engler, Introduction to Texture Analysis: Macrotexture, Microtexture, and Orientation Mapping, 2nd ed. CRC Press LLC, 2017.

[17] M. Varma and A. Zisserman, "A Statistical Approach to Texture Classification from Single Images," Int. J. Comput. Vis., vol. 62, no. 1/2, pp. 61-81, Apr. 2005.

[18] Abbas F. H. Alharan, Hayder K. Fatlawi, and Nabeel Salih Ali, "A cluster-based feature selection method for image texture classification," Indonesian Journal of Electrical Engineering and Computer Science, vol. 14, no. 3, pp. 14331442, June 2019.

[19] A. M. Hasan, "A Hybrid Approach of Using Particle Swarm Optimization and Volumetric Active Contour without Edge for Segmenting Brain Tumors in MRI Scan", Indonesian Journal of Electrical Engineering and Informatics, vol. 6, no. 3, , pp. 292-300, September 2018.

[20] H. Tamura, S. Mori, and T. Yamawaki, "Textural Features Corresponding to Visual Perception," IEEE Trans. Syst. Man. Cybern., vol. 8, no. 6, pp. 460-473, 1978.

[21] M. Niemeijer, J. Staal, B. van Ginneken, M. Loog, and M. D. Abramoff, "Comparative study of retinal vessel segmentation methods on a new publicly available database," Proc. SPIE 5370, Medical Imaging 2004: Image Processing, (12 May 2004); doi: 10.1117/12.535349

[22] M. Niemeijer, M. D. Abramoff, and B. van Ginneken, "Segmentation of the Optic Disc, Macula and Vascular Arch in Fundus Photographs," IEEE Trans. Med. Imaging, vol. 26, no. 1, pp. 116-127, Jan. 2007.

[23] R. Kohavi and G. H. John, "Wrappers for feature subset selection," Artif. Intell., vol. 97, no. 1-2, pp. 273-324, Dec. 1997.

[24] S. Srivastava, M. R. Gupta, and Béla A. Frigyik, "Bayesian Quadratic Discriminant Analysis," J of Mach. Lear. Res., vol. 8, pp. 1277-1305, 2007.

[25] R. O. Duda,. Peter E. Hart, and D. G. Stork, Pattern classification,2nd ed. Wiley, 2000. 
[26] L. Tarassenko and S. Roberts, "Supervised and unsupervised learning in radial basis function classifiers," in IEE Proceedings - Vision, Image and Signal Processing, vol. 141, no. 4, pp. 210-216, Aug. 1994. doi: 10.1049/ip-vis:19941324

[27] Kee Yong Pang, I. Lila Iznita, M. H. Ahmad Fadzil, A. N. Hanung, N. Hermawan, and S. A. Vijanth, "Segmentation of retinal vasculature in colour fundus images," in 2009 Innovative Technologies in Intelligent Systems and Industrial Applications, pp. 398-401, 2009.

[28] Wiharto Wiharto, Esti Suryani, and Vicka Cahyawati, "The methods of duo output neural network ensemble for prediction of coronary heart disease," Indonesian Journal of Electrical Engineering and Informatics, vol. 7, no. 1, pp. 51-58, March 2019.

[29] P.J.Navarro, D. Alonso, K. Stathis "Automatic detection of microaneurysms in diabetic retinopathy fundus images using the $L^{*} a * b$ color space," in Journal of the Optical Society of America, vol. 33 no. 1, pp. 74-83, June 2016.

[30] Arun Pradeep, X Felix Joseph, " Binary operation based hard exudate detection and fuzzy based classification in diabetic retinal fundus images for real time diagnosis applications," International Journal of Electrical and Computer Engineering, vol. 10, no. 3, pp. 51-58, June 2020.

\section{BIOGRAPHY OF AUTHORS}

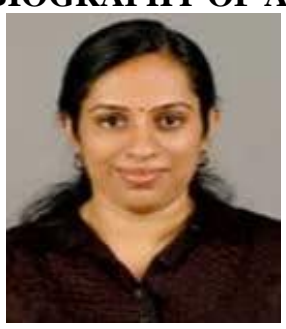

Sreeja K A Received B.Tech In Electronics and Communication and M.Tech in Embeddded Systems Technology. Currently she is working as Assistant Professor in Department of Electronics and Communication Engineering. SCMS School of Engineering and Technology, Karukutty, Ernakulam, Kerala. In past, from 2004 to 2007 she worked as Assistant Professor in Electronics and Communication at Sarabhai Institute of Science and Technology, Thiruvananathapuram. Her research interest area is Medical Image Processing.

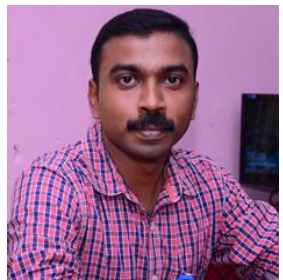

Kumar S S is currently working as the head of Department of Electronics and Instrumentation Engineering. NICHE, Thuckalay. Kanyakumari Dt. Tamil Nadu, India. In past, he worked as Associate Professor, Dept. of Electronics and Instrumentation Engineering, Noorul Islam University, Thuckalay. His research interest fields are Neural networks and medical image processing. 